\title{
Critical thinking and the methodology of science
}

\author{
Presentation to the \\ Australasian Science Education Research Association \\ Brisbane, Australia June 2021
}

\author{
Peter Ellerton \\ University of Queensland Critical Thinking Project \\ The University of Queensland \\ Australia
}

\begin{abstract}
Scientific literacy concerns understanding the methodology of science at least as much as understanding scientific knowledge. More than this, it also requires an understanding of why the methodology of science delivers (or fails to deliver) epistemic credibility. To justify scientific claims as credible, it is important to understand how the nature of our reasoning is embodied in scientific methodology and how the limits of our reasoning are therefore the limits of our inquiry. This paper makes explicit how aspects of critical thinking, including argumentation and reasoning, underpin the methodology of science in the hope of making the development of scientific literacy in students more actionable.
\end{abstract}

Critical thinking is not necessarily owned, or even best propagated, by any particular discipline. It does, however, find an academic home in philosophy. The reason for this is quite straightforward: questions of what makes for a good reason, why the answer to those questions should compel us to accept such reasons and even the nature of rationality itself are philosophical questions. In other words, philosophy provides a normative framework for understanding critical thinking.

This normative framework is a rigorous one, involving a range of skills, values and virtues. Core technical skills include those of argument construction and evaluation (and within these the use of analogy and generalisation), collaborative reasoning skills and communication skills including effectively linking good thinking and writing. Values such as accuracy, precision, simplicity, reproducibility, coherence and relevance and the ability to apply these are essential to critical and empirical inquiry. Inquiry virtues include open-mindedness, intellectual honesty and humility and the application of the principles of honesty and charity. Driving all this is the imperative to seek new possibilities, reinterpret old problems and question underlying assumptions.

This assortment of capabilities and characteristics is desirable across all areas of inquiry and is operationalised within each discipline through the methodology that is taught, practiced and critiqued through processes such as formal discipline education, collaborative investigations and peer review. In other words, critical thinking is baked into effective inquiry. 
This might seem to suggest that an education in discipline methodology will, of necessity, deliver an education in critical thinking, but we should be careful of such claims for a number of reasons.

The first reason is that a significant amount of discipline training at the undergraduate level focusses on analytical processes. Problems that are complex, challenging and demand a substantial breadth and depth of content knowledge are often seen as effective training grounds for critical thinking. This may be true in some cases, but analysis is not, of itself, critical thinking. If analysis is sufficient for critical thinking, then we must allow that computers can think critically since they perform many analytical tasks for us - but we do not make this allowance. Critical thinking, therefore, is not exhausted by, nor perhaps even represented by, the fact of complex analysis.

The second reason is that following a methodology does not imply an understanding of the methodology. The epistemic credibility of a claim within a discipline is only weakly made by appealing to the norms of inquiry of that discipline. A full justification would require an explanation of why an application of those norms grants credibility.

The third reason is that the explicit nature of the critical thinking skills within a discipline are not always the object of study of that discipline (interestingly, it is this characteristic that seems to distinguish philosophy from other discipline areas). It is the last of these reasons that grants us an insight into how education and practice of science can be improved through explicit instruction and experience in the use of critical thinking concepts and skills. Showing how a methodology of inquiry instantiates aspects of critical thinking and explaining why that is epistemically meaningful represents both a development in critical thinking skills and a progression towards inquiry virtues. It is not surprising, therefore, to occasionally hear critical thinking framed as 'applied epistemology'.

Science - as Thomas Kuhn (1970) notes - is not rule-based; instead, scientists preferentially apply values such as simplicity, clarity, plausibility and accuracy in the act of inquiry shaped to context and purpose. It also seeks collective virtue through reflective and adaptive processes including peer review and collaborative experimental inquiry. Mathematics finds epistemic justification through rigorous deductive reasoning and application through developing and critically testing models. Engineering and technology disciplines meld design, creativity and reflective practice to create teachable and translatable problem-solving process based on cognitive skills. How we understand the underlying thinking skills that make up these discipline methodologies is a critical factor in teaching students not just about the nature of STEM inquiry, but also in building their critical thinking skills. Let me now consider how this rational discipline structure can be made explicit.

In developing student cognition, we must ensure that students have opportunities for genuine doubt, for doubt is the reason for inquiry, and inquiry provides a reason for the use of cognitive skills. But doubt is not something to be allocated, it must be cultivated. As Matthew Lipman (2003) points out:

If, then, thinking in the classroom is considered desirable, the curriculum cannot present itself as clear and settled, for this paralyses thought. (p.21)

Science and mathematics curricula are typically content heavy and procedurally prescriptive, a logical outcome of centuries of successful empirical inquiry and of developing highly 
refined inductive and deductive methodologies. This knowledge load means that science and mathematics classes are susceptible to passive modes of student learning characterised by the controlled, high-volume flow of information from teacher to student. Doubt and inquiry have little traction or use in such modes.

The task of cultivating inquiry requires a rethink of curriculum design away from a focus on content-based delivery to genuine inquiry, but we should not fall into the trap of thinking that inquiry is exemplified, or even satisfied, by experimentation. If we understand good inquiry as that which demands of students a broad and deep range of cognitive skills, then a large number of structured investigations that algorithmically move students towards an inevitable conclusion, the pull of which is obvious even to them, are excluded. We should also not imagine that anything under the banner of 'problem-based learning' has any more claim to being good inquiry than the category of 'experiment', and for the same reasons. My focus for the moment, however, is not to analyse what makes for good inquiry in these approaches but is on how a better understanding of the methodologies (not just the practice) of science can cultivate genuine doubt and hence provide opportunities to develop thinking skills through inquiry. One way to do this is to examine how these methodologies are aligned to our means of reasoning and to recognise how they capture normative aspects of good thinking.

Mathematics and science are famously demarcated by how each discipline uses deduction and induction, the two categories of inference into which most of our reasoning falls.

Deduction is characterised by the adoption of certain axioms (the truth of which is either taken to be intuitively obvious or assumed for the purposes of the context) and the consequent discovery of propositions that can be logically derived from those axioms. A classical example of deductive reasoning includes Euclid's axiom that parallel lines never meet, from which are deduced many of the propositions of geometry.

In any deductive argument the truth of the premises guarantees the truth of the conclusion, which is logically derived from them. Such an argument is said to be valid. An example of a valid, deductive argument is:

\section{Premise one: All gronks are green \\ Premise two: Fred is a gronk \\ Conclusion: Fred is green}

In the above argument, if someone accepts the truth of the premises but does not agree that Fred is green, then that person has made a mistake in reasoning. Most people's experience with mathematics involves learning to reason deductively in this way. For example, consider the familiar mathematical problem below, expressed as an argument:

$\begin{array}{ll}\text { Premise one: } & y=m x+c \\ \text { Premise two: } & m=1 \\ \text { Premise three: } & x=2 \\ \text { Premise three: } & c=5\end{array}$

It follows deductively from these premises that $y=7$ (necessary to make the argument valid). To think otherwise is to make the same mistake as someone who thought that Fred was not green in the earlier example. 
The object of study of much of mathematics is to determine the legitimacy of logical inferences drawn from axiomatic positions, something that cannot be determined by empirical investigation. Whether all gronks are green and whether Fred is a gronk are certainly empirical claims, but the legitimacy of the inference we draw from them is not. It requires an appeal to the norms of deductive reasoning. Deductive reasoning of this sort is the typical cognitive experience of students in mathematics education. Since, however, deduction is less concerned with the truth of premises or conclusions than it is with the logical relationships between them, it can seem that the contexts of deduction are decoupled from practical concerns (after all, there are no such things as gronks). If the propositions themselves are not empirically grounded, then students might feel quite justified in asking "when are we ever going to use this?" (a question that is arguably best responded to by saying "whenever you are required to think deductively"). The strength of deduction is its certainty, but its weakness is its inability to extend empirical investigation. While we can deduce the hitherto unknown characteristics of a novel hypothetical space-time topography given certain axioms, we cannot deduce the lifespan of a tiger salamander - we must go and look at it for a while.

But mathematics education does not only focus on the rules of deduction. Much of what students experience in mathematics classes also involves modelling real world situations with mathematical equations. This modelling can extend into areas a diverse as economics, geology, biological systems and population dynamics. That systems of any sort can be described using mathematics is a profound fact about the universe, and there is no immediate reason why this should be possible. As the physicist Eugene Wigner noted in his famous essay The Unreasonable Effectiveness of Mathematics in the Natural Sciences (1960), "the enormous usefulness of mathematics in the natural sciences is something bordering on the mysterious and that there is no rational explanation for it."

One of the first things that students come up against in modelling individual situations, however, is that it always involves approximations and limitations of one sort or another. The reason that such approximations and limitations occur usually requires an understanding, or at least description, of the dynamics of the systems being modelled. A linear model may only be applicable over a certain range of variables, outside of which the system exhibits nonlinear behaviour, a broad trend modelled by a power curve fails to capture variability at high resolution, and so on. The creation and testing of mathematical models may be theory driven or data driven, but each moves away from deductive thinking and into inductive modes of reasoning wherein models produce analogous behaviour even though they themselves are not analogies. Let me consider this through the use of induction in science.

While deductive reasoning is limited to teasing out the logical implications of a given set of axioms, science is concerned with empirical investigation of the world and of the creation of new knowledge that describes it (not just knowledge that can be deductively derived from existing propositions). While some of this new knowledge comes from simple observation much of it is gained through inductive inferences based on observation and theoretical assumptions. Unlike deduction, inductive inferences do not preserve truth claims from premises to conclusions, in other words, the truth of the premises does not guarantee the truth of the conclusion. Consider the inductive argument below:

Premise one: Each time I've had a chocolate covered oyster I've been sick

Premise two: I've just had a chocolate covered oyster

Conclusion: I am going to be sick 
While the premises collectively make a strong case for the conclusion, it is not the case that the conclusion necessarily follows from the premises - it is possible I may not be sick. The argument is hence invalid, but that does not mean it is not rationally persuasive. The confidence we can have in an inductive conclusion requires further analysis of the situation and a need to justify why the conclusions are considered rationally persuasive, usually by recourse to additional information that bears on the credibility of the inference. This raises another important distinction between deductive and inductive arguments: deductive arguments are indefeasible, while inductive arguments are defeasible. Defeasible arguments are those whose conclusions can be strengthened or weakened by the addition of additional premises. Indefeasible arguments cannot be so modified.

Consider the inductive argument above with the addition of extra premises:

Premise one: Each time I've had a chocolate covered oyster I've been sick

Premise two: I've just had a chocolate covered oyster

Premise three: All the previous oysters I've eaten were kept in the cupboard

Premise four: The most recent oyster was kept in the fridge

Conclusion: I am going to be sick

The addition of premises three and four weaken the inferential connection to the conclusion, it is defeasible. Consider now the deductive argument from above with the addition of extra premises:

Premise one: All gronks are green

Premise two: Fred is a gronk

Premise three: All gronks are smelly

Premise four: It's not easy being green

Conclusion: Fred is green

Note that the addition of premises three and four have no effect on the strength of the inferential connection between the original premises and the conclusion. Indeed, no addition information can render the argument invalid (though contradictory premises may render it meaningless). It is in this distinction between indefeasible and defeasible arguments that we can meaningfully address the concept of proof in mathematics and science.

A common view of proof is of something that has been established beyond doubt or the possibility of doubt (though an older use of the term as of something having been trialled or tested is still with us, if only just, in the use of the term 'proving ground'). Proofs proliferate in mathematics and for good reason, they are creatures of deduction requiring the inferential certainty that only deduction can provide. Euclid's proof of the infinitude of prime numbers or the proof that the square root of 2 is irrational are deductively derived. Because they are deductive proofs (read deductive arguments), they are therefore indefeasible. No matter what else we find out, the proofs cannot be defeated by the addition of extra premises.

Mathematical proof gives us proof for the ages. The concept of scientific proof, however, is more nuanced. Since science works primarily through induction, to say that something is 'scientifically proven' is often an appeal to something other than inferential certainty. Appeals to proof in science rest on three broad platforms. The first is confidence in direct observation, the second is in the concept of falsification through deductive inference and the third is the strength of an analogy or generalisation. Let me very briefly address these in turn, 
with the intent of providing enough background to indicate the value of the topic to cultivating doubt and inquiry.

Direct observation of nature is common to all of us. We do not spend much time arguing about general experiences regarding the colour of a clear daytime sky, whether or not the sun can provide warmth, or whether or not wood is flammable. We could 'prove' any of these claims quite easily through simple experience. But we should be very careful about appeals to experience, after all the earth does not seem to be spinning and looks flat from where we usually stand. It is appeals to exactly these kinds of common experiences (and intuitions) that drives much of pseudoscience. Indeed, science may well be considered a defence against our 'common sense' ideas and perceptions.

We might also seek proof through falsification and it is here perhaps the strongest case can be made, at least in terms of deductive thinking, for falsification requires a deductive argument. The philosopher Karl Popper (1959), dissatisfied that inductive reason could be properly justified, proposed placing all our rational scientific eggs in the basket of the hypotheticodeductive method. The thinking goes like this. A hypothesis is generated, by whatever means, which makes a prediction regarding some experimental outcome. The experimental conditions are provided, but the expected outcome is not observed. The conclusion can be reached deductively that the hypothesis is not correct. In other words, the hypothesis is falsified. The argument looks like this:

Premise one: If my hypothesis, $\mathrm{H}$, is true then, under condition $\mathrm{X}, \mathrm{E}$ will occur Premise two: Condition $\mathrm{X}$ is provided

Premise three: E does not occur

Conclusion: $\mathrm{H}$ is false

Put symbolically and more simply

$$
\begin{aligned}
& \mathrm{H} \rightarrow \mathrm{E} \text { (if } \mathrm{H} \text { then } \mathrm{E}) \\
& \text { Not } \mathrm{E} \\
& \therefore \operatorname{not} \mathrm{H} \text { [valid] }
\end{aligned}
$$

Let us accept, for the moment, that this can constitute a proof of the sort we attribute in mathematical contexts. What is it that we have proved? It is not that the hypothesis is true, but that it is false. If we attempt to articulate an example of a positive outcome, we get the argument below:

Premise one: If my hypothesis, $\mathrm{H}$, is true then, under condition $\mathrm{X}, \mathrm{E}$ will occur

Premise two: Condition $\mathrm{X}$ is provided

Premise three: E does occur

Conclusion: $\mathrm{H}$ is true

$$
\begin{aligned}
& \mathrm{H} \rightarrow \mathrm{E} \\
& \mathrm{E} \\
& \therefore \mathrm{H} \text { [invalid] }
\end{aligned}
$$

This argument, however, is an example of the fallacy of affirming the consequent. The argument is invalid. The conclusion is not necessarily true given the truth of the premises. While falsification through the hypothetico-deductive method can be deductively valid, it can 
only apply to what is not the case, not what is the case. In an ironic sense, through falsification science can only prove a negative. It is also in this deductive structure (the conditional statement that is premise one) that we get the language of necessary and sufficient conditions, i.e. $\mathrm{E}$ is necessary to say that $\mathrm{H}$ is true, and $\mathrm{H}$ is sufficient for $\mathrm{E}$. But even were we willing to make do with certainly only in the rejection of hypotheses rather than the acceptance of them, we cannot whole extricate ourselves from inductive concerns. As many critics of falsification have pointed out, no hypothesis is ever tested in isolation, it belongs to a great web of supporting hypotheses that make up a large body of often unquestioned assumptions. If $\mathrm{E}$ does not occur, we cannot know for certain where in this web of assumptions our belief is misplaced, since it might be in a supporting hypothesis rather than in $\mathrm{H}$ itself. Moreover, the experimental conditions that supposedly make up condition X might also be in error. Famously, the Oscillation Project with Emulsion-tRacking Apparatus (OPERA) experiment of 2011 detected neutrinos that seemed to travel faster than light, in defiance of the predictions of special relativity. Scientists did not assume that special relatively was falsified, but sought, successfully as it turned out, for a source of experiment error to explain that anomaly.

Inductive reasoning in science is of two general sorts, analogy and generalisation. We reason by analogy when we seek similarities between two cases and take the conclusion drawn from one as justification to draw the same conclusion for the other. As an example, consider a palaeontologist who discovers the complete fossilised skull and jaws of an extinct creature. Imagine that the jaws have large, prominent, pointed teeth. The palaeontologist might conclude that the animal was a carnivore, but what justifies this claim is analogous reasoning. Since we find that other animals with similar tooth structures alive today are carnivores, we transfer that finding to this case, an inductive conclusion but one in which we nevertheless have confidence. The more similarities we find with the fossil and existing carnivores, including the structure of other teeth in the jaw and the apparent musculature around the skull (more analogous reasoning required there), the more confident we are in our conclusion. A later finding that at least two species of herbivorous deer, the Chinese water deer and the musk deer, also have fang-like teeth would weaken our analogy, however, reminding us that inductive reasoning is defeasible. Reasoning by generalisation involves extending claims about a number of members of a set to all members of the set. The justification for this extension includes that the number of members of the set, the sample, is sufficiently large and that there is sufficient variety within the sample. Generalisation does not only justify claims such as 'all koalas are grey' or 'all gronks are green', it also provides justification for the laws of physics. For example, we note that in all cases so far seen that the gravitational attraction between two bodies is proportional to the inverse of the square of the distance between them. From these cases we generalise to the universal claim that all bodies behave the same way (ceteris paribus) and derive the inverse square law of gravity. But we should consider many things about the so-called laws of nature, including whether the regularity being observed is necessarily that way or simply contingent within our experience, whether it is a regularity that is suitable for generalisation (pigeons wearing hats on university grounds is better explained by university parties than understood as a fixed property of pigeons) or whether we are operating within a paradigm that allows the generalisation to be accurately expressed (are all objects combinations of the four elements, as the ancient Greeks once thought?).

The distinction between a theory and a law is drawn from the distinction between analogies and generalisations. Our theories are designed to be analogous to the universe, or parts of it. We judge the quality of a theory by how analogous its inputs and outputs are with that of the 
real world. The more alike they are, the more confident we are that we are on the right track (whether or not that confidence is justified is another question). Our laws are generalisations of behaviours that suggest regularities in nature. Laws also describe regularities, but they do not explain them. Theories do not become laws over time any more than analogies become generalisations. Theories and laws do not represent different levels of epistemic credibility. The deeply mistaken belief that theories become laws once they have withstood some arbitrary level of testing is at the core of much of the public misunderstanding of science. If theories did develop into laws, it would not have been possible to have Newton's law of gravity before Einstein's theory of general relativity, a clearly absurd prospect. Indeed, theories often develop in order to explain apparent laws, providing explanatory frameworks that help understand why the regularity occurs.

The relationship between methodology and reasoning in science is summarised in Figure One.

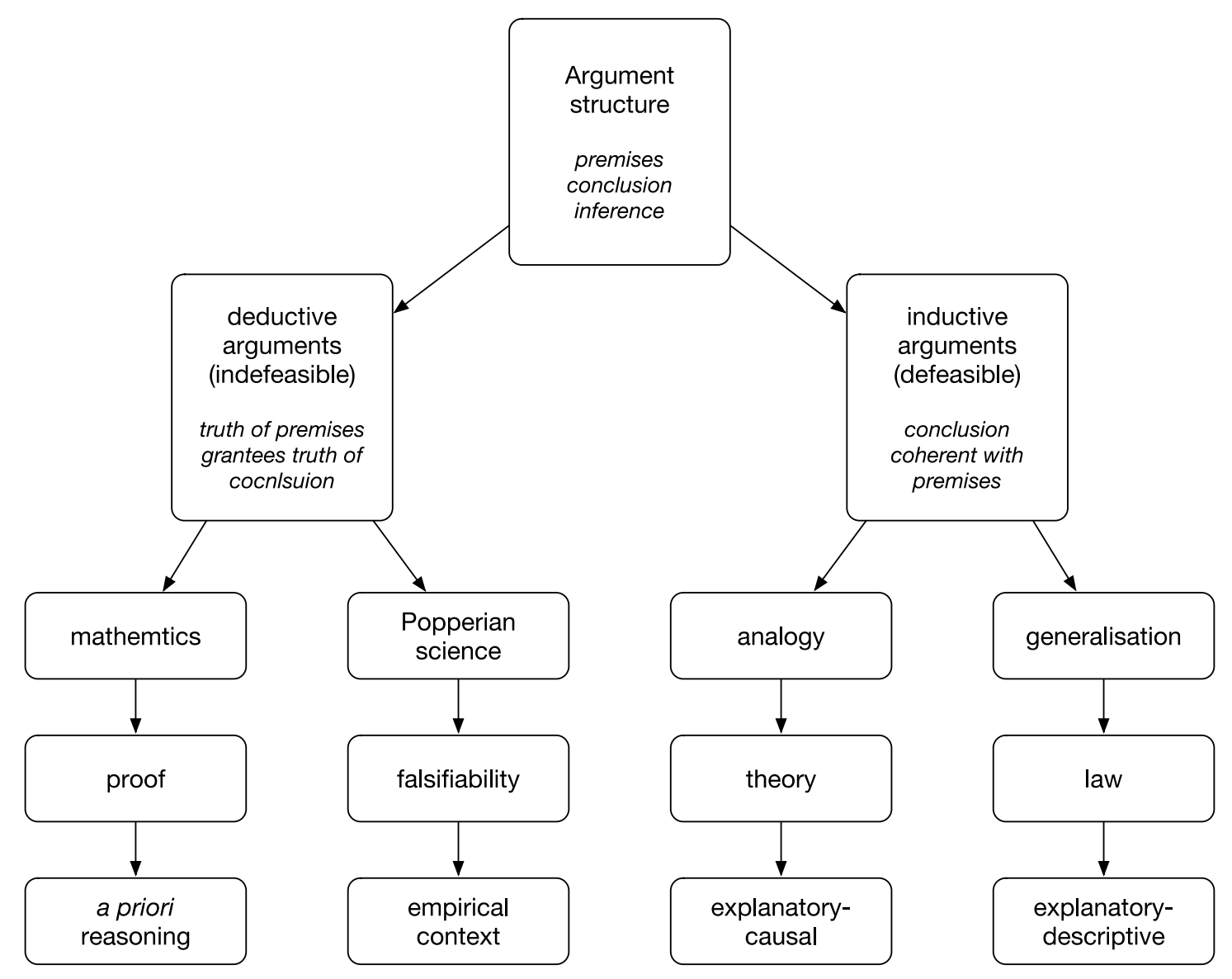

Figure One: reasoning embedded in scientific methodology

\section{Conclusion}

Science use mathematical modelling and reasoning by induction for instrumental ends and for the purposes of discovery. The study of the legitimacy of deductive moves is not usually a requirement of science. Science does, however, also involve reasoning deductively through mathematics and when using conclusions from inductive arguments as premises (for 
example, that all gronk are green). Whether we are reasoning deductively or inductively, the quality of our inquiry is a function of the quality of our thinking - the kind of thinking embedded in discipline methodology. Students, therefore, need to be able to not only recognise and use discipline thinking, but also to evaluate it. Making this thinking explicit, and teaching students how to analyse and evaluate it, is key to developing scientific literacy, which must extend beyond simply knowledge of scientific content or even the understanding of scientific concepts. Another fundamental aspect of scientific literacy is understanding inquiry as the means of production of knowledge. Inquiry in science education is encouraged when students can engage in genuine doubt. But this doubt is not simply a matter of pretence or form. As the philosopher C.S. Peirce puts it, "the mere putting of a proposition into the interrogative form [i.e. asking a question] does not stimulate the mind to any struggle after belief. There must be a real and living doubt, and without this all discussion is idle".

Understanding the reasoning inherent in a methodology not only highlights its credibility as a means of inquiry but also exposes why some of its claims will remain forever tentative. Students must see that the limits of empirical inquiry are not separable from the limits of rational inquiry. In this realisation there is the opportunity for genuine doubt and a recognition of the need for evaluating inquiry.

\section{References}

Kuhn, T. S. (1970). The Structure of Scientific Revolutions. In International Encyclopedia of Unified Science (2nd ed., Vol. 2). Chicago: University of Chicago Press.

Lipman, M. (2003). Thinking in Education. Cambridge: Cambridge University Press. doi:10.1017/CBO9780511840272

Peirce, C. S. (1877). The Fixation of belief. Popular Science Monthly, (12), 1-15.

Popper, K. (1959). The logic of scientific discovery. London: Hutchinson.

Wigner, Eugene (1960). The Unreasonable Effectiveness of Mathematics in the Natural Sciences. In Communications in Pure and Applied Mathematics, vol. 13, No. I. New York: John Wiley \& Sons, Inc. 